\title{
Are Radio Pulsars Extraterrestrial Communication Beacons?
}

\section{LaViolette PA*}

The Starburst Foundation, 1176 Hedgewood Lane, Niskayuna, NY, 12309, USA

*Corresponding author: LaViolette PA, The Starburst Foundation, 1176 Hedgewood Lane, Niskayuna, NY, 12309, United States, Tel: +1 518-372-2711; E-mail: plaviolette@starburstfound.org

Received date: Feb 08, 2016; Accepted date: March 01, 2016; Published date: March 08, 2016

Copyright: ( $\odot 2016$ LaViolette PA. This is an open-access article distributed under the terms of the Creative Commons Attribution License, which permits unrestricted use, distribution, and reproduction in any medium, provided the original author and source are credited.

\begin{abstract}
Evidence is presented that radio pulsars may be artificially engineered beacons of extraterrestrial intelligence (ETI) origin. It is proposed that they are beaming signals to various targeted Galactic locations including our solar system and that their primary purpose may be for interstellar navigation. More significantly, about half a dozen pulsars appear to be marking key sky locations that convey a message intended for our Galactic locale. One of these, the Millisecond Pulsar (PSR1937 + 21), appears to make reference to the center of our Galaxy, which would be a logical shared reference point for any interstellar communication. It is noted that of all pulsars, this one comes closest to the point that lie one-radian from the Galactic center along the galactic plane. The chance that a pulsar would be positioned at this key location, as seen from our viewing perspective, and also display the highly unique attention-getting characteristics of the Millisecond Pulsar is estimated to be one chance in 7.6 trillion. Other pulsars that appear to be involved in conveying this Galactic center message include the Eclipsing Binary Millisecond pulsar $(1957+20)$ and PSR $1930+22$, both of which make highly improbable alignments relative to the Millisecond Pulsar position, the Crab and Vela pulsars, and PSR $0525+21$. All display one or more unusual attention-getting characteristics. A method is proposed whereby a civilization might magnetically modulates the cosmic ray flux of a neutron star to produce one or more stationary, broadband, targeted synchrotron beams having pulsar-like signal characteristics. Also a lower tech approach is proposed that would instead modulate the relativistic electron beam from a linear particle accelerator to produce a pulsed free electron laser beam.
\end{abstract}

Keywords: Radio pulsars; Extraterrestrial; Beacons; Panspermia; Protobionts; Galaxy; Galactic center; Gamma sagittae; Gyrotrons; Cosmic rays

\section{Introduction}

The following intends to demonstrate that most or all radio pulsar signals are likely of artificial origin, hence challenging the standard assumption that these signals arise naturally. Here, a distinction should be made between the neutron stars, which provide the cosmic ray energy powering pulsar signals which are of natural origin, and the pulsar signals themselves which I suggest are purposely engineered by manipulating an existing stellar cosmic ray flux for purposes of communication.

While the same name, "pulsar," is used to refer to all types of pulsars, it is advisable to distinguish here between the radio-quiet Xray and gamma ray pulsars and those that belong to the radio pulsar category. Since the first category do not share the highly ordered characteristics of radio pulsars, they indeed are likely of natural origin. Their pulsed signals can be adequately explained by means of Thomas Gold's lighthouse model or some variation of it. That is, such sources may be modeled as being cosmic-ray-emitting neutron stars whose cosmic ray flux interacts with a strong polar magnetic field causing the star to beam synchrotron or bremsstrahlung radiation from one or both of its poles. As the star rotates, it periodically flashes its X-ray (or gamma ray) beam in our direction creating a continuous train of pulses.

Examples of such pulsars include the matter accreting millisecond $\mathrm{x}$-ray pulsars and slow pulsars found in accreting X-ray binaries such as those reviewed by Patruno and Watts [1]. The well-known $\mathrm{x}$-ray pulsar Hercules X - 1 is an example of a pulsar that is admittedly a natural source. Generally the integrated X-ray or gamma ray pulse profile of such sources has noticeable random variation, making the profile's shape and timing rather imprecise. Adding up successive pulses produces an integrated profile that still embodies a significant amount of random variation. Such timing variability is what one would expect if one were observing a synchrotron-emitting neutron star that was producing electromagnetic emissions through natural processes. Moreover pulsars in this class normally are not seen to have a radio counterpart to their pulses. Radio emission, if observed, is seen during transient flare events associated with matter accretion episodes and is assumed to come from a radio jet associated with the accretion event; for example, see Jonker et al. [2].

Radio pulsars, on the other hand, seem to be an entirely different type of phenomenon. Since the time they were discovered, they hold the unique status of being the most complex and most highly ordered phenomenon known to astronomy. Besides exhibiting very high precision in the timing, shape, and polarization of their integrated pulse profile, they also exhibit a variety of subpulse and pulse profile ordering characteristics which give each pulsar additional unique features in their signal signature. Some of these ordering characteristics are listed in Table 1 .

- Subpulse amplitude modulation: subpulses oscillate in amplitude with a characteristic period P3 different from the pulsar's pulse profile period P1, and this pulsation in some pulsars is seen in every pulse and in other pulsars is seen in every other pulse.

Subpulse phase drifting: subpulses can drift in phase across the pulse profile with a charactistic period P3 different from $\mathrm{P} 1$, drifting either from 
back to front or front to back, at either a constant rate, a decelerating rate or an accelerating rate.

- Multicomponent correlated subpulse phase drifting: pulse profiles having multiple components can exhibit subpulses in all components that drift at the same rate in synch with one another, in some pulsars their drifting can reverse direction at random times, in others the drift rate increases with each successive pulse until it reaches a threshold where its drift direction reverses and the rate slows down with each successive pulse.

- Nonrandom complex pulse patterns: Subpulse patterns consisting of three to six successive subpulses occasionally recur even though no subpulse drift paths are traceable.

- Pulse profile mode shifting: the pulse profile can alternate between two to four different modes, each characterized by a different pulse profile shape, polarization pattern, and subpulse oscillation or drift phenomenon. Which modes are observed and the character of each can change depending on the particular radio frequency the pulsar is observed at.

- $\quad$ Pulse profile mode shifting with quantization of subpulse drift rate: When the pulsar switches between alternate modes, subpulse drift rate can change in steps of two times or three times the base drift rate.

- Pulse profile mode switching grammar: Of modes $A, B$, and $C$, only modes $A$ and $B$ or modes $B$ and $C$ appear together in a given pulse burst, modes $A$ and $C$ never appear together.

- Pulse profile mode switching with mode memory: Pulsars that have mode switching rules that govern how they switch their modes "remember" their previous mode from a previous pulse burst to determine how they switch in the subsequent burst.

- Sporadic nulling of the pulsar's emission and drift memory: A pulsar can suddenly shut off its pulsing at random intervals for varying lengths of time. When it turns on again it "remembers" its previous pulse drift phase and continues its pulse drifting where it left off.

Table 1: Varieties of pulsar ordering characteristics.

These ordering characteristics are so varied and unique that efforts to model them have proven futile. For example, radio astronomers Filippenko and Radhakrishnan [3] state:

"The standard polar cap model of pulsar radio emission provides acceptable explanations for a wide variety of observed pulsar characteristics. Nevertheless, we show that it has difficulty accounting for certain details pertaining to drifting subpulses, nulling, and mode changing. In particular, the persistence of drifting subpulse phase memory observed during pulsar nulling, as well as the phenomenon of nulling itself, seems to defy simple explanation."

Radio astronomers Bartel et al. [4] are also perplexed about modeswitching phenomenon in radio pulsars. They write:

"Despite the fact that the mode-switching phenomenon has such an important effect on pulse emission, no elaborate theory as yet exists as to how to interpret it."

Radio astronomers Taylor et al. [5] also admit the confused status of this area of astronomical research. They state:

"There exists an extensive literature of observational details which, although well substantiated by repeated measurements, remain unexplained and unassimilated into pulsar models."

Pulsar astronomers, Gil et al. [6] state:

"Although almost 30 years have passed since discovery of pulsars, their radiation still remains a mystery. This concerns both the fundamental problem of the coherent radio emission as well as the specific modulation of pulsar radiation in the form of individual pulses and the characteristic stable mean profiles."

More recently, pulsar astronomers, Gil et al. [7] state:

"40 years after discovery of pulsars the actual mechanism of their coherent radio emission is still a mystery. Drifting subpulses, which seem to be a common phenomenon in pulsar radiation, is also a puzzle."

This modeling difficulty continues to be a problem even today, e.g., as seen with the discovery that mode switching pulsar PSR $0943+10$ exhibits abrupt synchronous switching of its radio and X-ray emission with X-ray emission being strongest when radio emission is weakest [8]. Also a paper by Andersson et al. [9] overturns a 40 year old theory that attempts to explain the phenomenon of period glitching leaving a natural cause explanation for the phenomenon to be an open question. It is apparent that radio pulsar signals are so complex as to elude all efforts to model them in a natural way as emissions produced by a rotating neutron star. As a result, of all astronomical objects, they are the best known candidates for being considered astro-engineered beacons of intelligent origin.

I began studying radio pulsars in 1979 , at a time when only a few hundred were known. At that time I had come to conclude that they were likely of ETI origin. To date the number of radio pulsar discoveries has grown to over 2400 and these new discoveries appear only to further support the conclusion I had come to back in 1979. I propose that their main function is to serve as navigation beacons for interstellar travel $[10,11]$. Collectively they may serve as a kind of galactic GPS system, allowing space travelers to triangulate their spacecraft's position during interstellar flight. Pulsar signals could be used not only to triangulate one's position in the Galaxy, but also to determine one's relative travel velocity and even to correct for any time dilation that might occur in cases where travel speeds might reach a substantial fraction of the speed of light. Others later independently suggested thoughts along similar lines, those future terrestrial space travelers, for example, might use X-ray pulsars for interplanetary and interstellar navigation.

Past publications have proposed that a small subset of the known radio pulsars, about half a dozen, may be conveying a message specifically intended for us $[11,12]$. Some observations about the sky positions of a few of these unique message-conveying pulsars are summarized in the sections that follow. As is shown, the evidence is difficult to explain in any way other than by assuming that pulsars are artificial beacons.

\section{Our Assumptions about Extraterrestrial Intelligence: Are They Too Restrictive?}

In arriving at this ETI interpretation, it is best not to be fettered by restrictive, preconceived notions about the possible technological advancement of extraterrestrial life, for example, the assumption that if intelligent beings live elsewhere in the Galaxy that they should not be much more advanced than we are. Or, the assumption that, because our own civilization does not intercommunicate with extraterrestrial civilizations (at least openly), that one should not expect there to exist multiple civilizations in the Galaxy that intercommunicate with one another, visit one another, or even be mutually organized among themselves in some manner.

The process of interpreting whether or not a deep space signal is of intelligent origin is difficult to separate from the assumptions that we, 
the message recipients, hold about life possibly existing elsewhere in our Galaxy. Take as an example the sequence of events that surrounded the first discovery of pulsars. The first radio pulsar was discovered in July 1967 by the British radio astronomy graduate student, Jocelyn Bell. This source recorded in her data initially attracted her attention because of it produced unusually regular broadband radio pulses, something not seen before in modern astronomy. When she and her Cambridge University research team combined successive pulses from this source to compose an integrated pulse profile, they found that the source's pulse repetition rate was exceedingly precise and that the shape and polarization pattern of its pulse profile was highly invariant. Because astronomical sources do not exhibit such precision, they initially became convinced that they had discovered what could very well be a message being sent from a civilization elsewhere in our Galaxy. Her research team, led by Professor Anthony Hewish, later labeled the radio source LGM-1, the initials being jokingly chosen as an abbreviation for "little green men".

As their radio observations continued, the Cambridge group later discovered additional pulsars which they labeled LGM - 2, LGM - 3, and LGM - 4. But at this point, with so many sources of similar character being discovered in different parts of the sky, doubts began to emerge, and after some deliberation, they chose to reject their initial hypothesis of this being a source of artificial origin. The reason they gave for their reassessment was that they felt it was highly unlikely that more than one civilization would be transmitting a message to us at the same time. Also they did not believe that different civilizations would use a similar transmission method, one utilizing a broadband radio synchrotron emission beam to produce its pulses. As a result, they concluded that the sources, although highly unusual, must be a natural phenomenon. So efforts were begun to devise stellar models that would account for the signals in natural terms, and this natural source interpretation has stuck ever since.

In rejecting the extraterrestrial intelligence (ETI) hypothesis, the Cambridge astronomers were in effect adopting the anthropomorphic assumption that a communicating ETI civilization would be technologically similar to our own, that they probably would not have the ability to travel far from their home planet and that they would be acting alone in their communication effort to make contact with civilizations outside their solar system. Based on this assumption, they saw the probabilities were very small that more than one independently acting civilization would be making contact with us at this particular period of time.

Now, half a century later, with the advancement of our own technology and with our discovery that stars in the Galaxy are more often than not orbited by planets, many of which could sustain life, our views about the possibility of extraterrestrial life have changed. The idea that there might be many advanced civilizations in the Galaxy, interacting, intercommunicating, engaging in interstellar space travel between their worlds, cooperating with one another, and possibly even organized into a kind of "Galactic federation" does not sound so farfetched to many astronomers any more. If pulsars had been discovered today, rather than 47 years ago, the ETI interpretation may not have been so readily rejected. This example shows us that there is in fact an inherent psychological dimension or interpretive bias embedded in the search for extraterrestrial intelligence.

If one chooses to interpret the pulsar findings presented here and elsewhere [11] in an ETI context, one is inevitably led to adopt a set of assumptions about the nature of Galactic extraterrestrial intelligence. These assumptions, which are listed in Table 2, differ radically from those that were held by the Cambridge University discoverers years ago.

- That there are a multiplicity of advanced civilizations inhabiting our Galaxy that intercommunicate with one another, that a large number of these are transmitting signals to us at this point in time and are using the same or similar method for interstellar communication.

- That these communicating extraterrestrial civilizations engage in interstellar travel. That is, they are not merely residents in their own planetary system, but voyagers as well, capable of traveling to other stars in the Galaxy. So, in this example we are assuming that they are not homebound like us, but have reached a more advanced stage of development.

- That these communicating extra-terrestrial civilizations have technologies that allow them to send superluminal communications across the Galaxy (possibly by a mode other than pulsar transmissions) and are capable of deep space interstellar travel at near luminal speeds to star locations hundreds of light years or more distant from their home star system.

- That civilizations living in differing parts of the Galaxy are organized into a "Galactic Federation" and cooperate with one another to orchestrate a message sent from various Galactic locations so that when these various communication beacons are considered together by the recipient civilization they are seen to convey a message with a particular meaning. For example, a series of communication beacons might be located at specific Galactic locations which when considered together convey a particular geometrical meaning or exhibit alignments that are difficult to explain in terms of random arrangements.

- That the communicating civilization (or civilizations) has developed the ability to engineer the cosmic ray flux from a neutron star so as to produce a series of nonrotating, broadband, synchrotron emission beams targeted at specific Galactic locations and have the ability to modulate these beams so as to produce pulsar-like radio emission pulses.

- That the communicating civilizations would likely make reference to the Galactic center (GC) in the message they direct to us, the GC being the Galactic location that all civilizations share in common and hence would be a likely reference point in an interstellar communication.

- That communicating civilizations make use of geometric relations in interstellar communications they are directing towards us, geometry being a universal language that should be known to any advanced civilization.

Table 2: Some assumptions about the existence of intelligent life in the galaxy.

It is not necessary to consider these seven assumptions or hypotheses as being proven in advance. Instead, consider them as a context which allows one to better understand the pulsar- ETI interpretation, namely the suggestion that radio pulsars may have an artificial origin, and that a subset of these may be intentionally communicating a message to us. It is useful to present the following analogy. Suppose that a forester has planted a forest of pine trees in a series of rows where the trees in a given row are not necessarily equally spaced. An uninformed observer looking at this forest for the first time from an arbitrary viewing perspective will think that he is seeing a pine forest that grew by natural means. But when the person is allowed to walk perpendicular to the rows, he will periodically adopt a viewing angle that allows him to see that the trees grow in regular rows. In the case where all trees are exposed to the same soil conditions and rainfall, the chances of the pines adopting such a highly ordered pattern by natural means is vanishingly small. However, by knowing about the possibility of tree farming, our observer is able to adopt a new perspective and to come to the more reasonable conclusion that this forest has been engineered, that the trees were originally planted in this fashion by a forester. 
So, let us think of radio pulsars as the trees and the unusually regular tree rows as corresponding to the unusual positional locations exhibited by the subset of unique pulsars we will be discussing. As in the case of the planted tree forest, the chance that these positional locations arose due to a chance of nature will be shown to be vanishingly small. So, think of the assumptions listed in Table 2 as a conceptual context that allows an ETI interpretation of radio pulsars appear more plausible.

Assumption (1), the possibility that a multiplicity of technically advanced civilizations may exist throughout the Galaxy, appears likely in view of recent findings which indicate that as many as 7 billion habitable planets may exist throughout the Milky Way and that of these as many as 40,000 may harbor technically advanced civilizations [13]. Also a plausible gene-enzyme hypercycle mechanism has been proposed by the author [14] which indicates the likelihood that selfreplicating protobionts may have spontaneously emerged from an amino acid and nucleo-base-rich primordial soup pre-existing on an earth-like planet. Primitive cellular life that first arose on one of the billions of earth-like planets residing in the Galaxy could have traveled through interstellar space to inseminate the primordial soups of other planets, in accordance with the theory of panspermia $[15,16]$. Cosmic ray and gamma ray volleys from recurring galactic core explosions impacting solar systems throughout the Galaxy would have greatly accelerated the rate of genetic mutation as well as sped up the evolution of higher life forms through an ongoing process of quantum speciation [17].

Of the assumptions listed in Table 2, assumption (3) may raise concerns for those who maintain that nothing can exceed the velocity of light. Contrary to the opinion of relativists, one can point to a number of laboratory experiments that unquestionably demonstrate the reality of superluminal signal propagation. One example is an experiment performed by Carôt et al. [18] which is an update of the experiment performed by Enders and Nimtz [19]. In 2012, demonstrating their technology to a conference room of about 20 physicists and mathematicians, Nimtz and Carot [20] sent microwave signals through a 50 meter-long copper tube waveguide at many multiples of the speed of light. Another example of superluminal propagation is an experiment performed by Obolensky in 2005 in which Coulomb ground waves produced by a high-voltage shock discharges were measured to travel across the laboratory at superluminal speeds with initial departure speeds as high as $5 c$; for a summary see discussion by LaViolette [21]. Another very interesting example is the experiment conducted by Podkletnov and Modanese [22] in which a high voltage pulse discharged from a superconducting anode was observed to produce a collimated gravity impulse wave whose speed was measured at $64 \mathrm{c}$. In other experiments these researchers measured gravity impulse speeds in excess of several thousand $\mathrm{c}$ [21].

The one thing that all these technologies have in common is that they generate longitudinal waves. So it is conceivable that technologies could be developed to send and receive such longitudinal wave transmissions across interstellar distances in support of the suggestion that ETI civilizations may intercommunicate superluminally. Also Podkletnov and Modanese [23] have demonstrated that their longitudinal impulses are able to gravitationally accelerate a variety of target masses, which means that such a technology potentially might be employed to propel a vehicle to near (or maybe even beyond) luminal speeds [21].
Assumption (5), the idea that a civilization might engineer the cosmic ray emission of a neutron star to produce a series of broadband synchrotron communication beams targeted on specific Galactic locations, was first suggested in 2000 [10-12], and is summarized in Sections 7 and 8 . Such a civilization would be ranked as a Kardashev, type II civilization. Other SETI researchers have also suggested the idea that the emission from a natural stellar source might be engineered for the purpose of interstellar communication. For example, a team of astronomers, calling themselves the "Stellar Lighthouse group", plan to study stars in a particular field of the sky which are cataloged in the Kepler data base to look for unusual stellar emission patterns that might indicate evidence of intelligent communication [24]. Their data base, though, consists entirely of optically visible stars. Hence their study will not be examining neutron stars or radio pulsars.

The Stellar Lighthouse group likely plans to establish a priori what characteristics they would consider as constituting evidence for an astro-engineered beacon and what statistical criteria they will use before beginning to conduct their analysis of the Kepler data set. This, of course, is a good approach. Pulsars, however, were not discovered as part of a SETI search. There was no program set up beforehand having the objective of detecting pulsed broadband radio sources with the aim of determining whether there might be sources with signal characteristics indicative of alien intelligence or whether such sources might be showing alignments with key Galactic benchmarks. The first radio pulsar was discovered through serendipity and subsequent discoveries came about simply to discover whether similar sources might exist elsewhere in the Galaxy.

\section{The Millisecond Pulsar: A Possible Astro-Engineered Communication Beacon}

Let us for the present suppose that civilizations exist in the Galaxy that are capable of deep space interstellar travel at near luminal speeds to star locations distant from their home star system and that they have the ability to engineer the cosmic ray flux of a neutron star so as to produce one or more collimated synchrotron radio beams directed towards a particular locale (Assumptions (2) and (5)). Also let us suppose that this group of communicating civilizations would likely make reference to the location of the Galactic center (Assumption (6)). Carl Sagan and Frank Drake, for example, saw fit to do so in their design of the Pioneer 10 space plaque message which was intended for communication to other civilizations. Consider then that if a group of intercommunicating civilizations scattered throughout the Galaxy did have the ability to place a communication beacon anywhere in the Galaxy and they wanted to communicate specifically with our star system, where would it be logical for them to place a beacon or beacons that would make explicit reference to the Galactic center as seen from our viewing perspective?

It would be unlikely that they would place their marker beacon in exact coincidence with the GC sky position as seen from our particular viewing direction since the strong broadband radio flux emanating from the Sagittarius $A^{\star}$ radio source would mask any such beacon, making it very difficult to detect. An off-center location would be a more likely choice. In fact, between 2002 and 2004, one very unusual bright transient pulsed radio source, GCRT J17445 - 3009, was observed at a sky position about $1^{\circ}$ of arc from the GC. This unique object is discussed further in Section 6.

One alternative location would be to place their communication beacon at the galactic anticenter location $(\ell=179.94, \mathrm{~b}=+0.05)$ 
where interference from background radio emission would be particularly low. When examining a region around the galactic anticenter, the pulsar that comes closest to this position is PSR J0538+ 2817. It lies $1.75^{\circ}$ away at galactic coordinate $\ell=179.72, \mathrm{~b}=-1.69$. Significantly, its longitude coordinate lies just $0.22^{\circ}$ from the anticenter longitude. It is found to be embedded in a 105 year old supernova remnant $S 147$ and is believed to have originated from the supernova explosion site at the remnant's center located at $\left(\ell=180.0^{\circ}, \mathrm{b}=-1.7^{\circ}\right)$. Hence to within the accuracy of knowing the remnant's center, this supernova explosion took place right on the galactic anticenter longitude coordinate, although the explosion site deviates $1.7^{\circ}$ in latitude. While it is within normal chance probability to find a pulsar lying within $1.75^{\circ}$ of the galactic anticenter, it is less probable to find one having a longitude coordinate deviating by less than a quarter of a degree from the anticenter longitude. At the time of writing, there are over 2400 known pulsars and statistics show that about $5 \%$ of these lie within $\pm 5^{\circ}$ of the galactic plane in the outer part of the Galaxy spanning the longitude range from $\ell=90^{\circ}$ through $180^{\circ}$ to $270^{\circ}$. So on the basis of chance occurrence, there should be odds of one in 7 that a pulsar would be located within $0.22^{\circ}$ of longitude of the galactic anticenter longitude; i.e., $(0.22 / 180) \times 2400 \times 5 \%=0.14$. This is not an astounding statistic, but it does catch one's attention, even considering that this pulsar has no unusual distinguishing characteristics that would cause us to want to single it out from the other pulsars.

Another alternative way of making reference to the Galactic center would be to mark its position along the galactic equator at northern or southern longitudes using the geometrical concept of the radian. Looking down on the galactic plane and representing the galactic equator as a circle with the solar system at the circle's center and the galactic center (GC) as a point on the circle's circumference at galactic longitude $\ell=-0.0558^{\circ}$, we may take a chord equal to the circle's radius and mark off two arcs on either side of the GC point, each one-radian in extent, hence deviating from the GC point by the angle $360^{\circ} / 2 \Pi=$ $57.2958^{\circ}$ (Figure 1). The Galactic center location may in this way be indirectly indicated by galactic longitude positions lying on either side of the GC at $\ell=302.648^{\circ}$ and $\ell=57.240^{\circ}$.

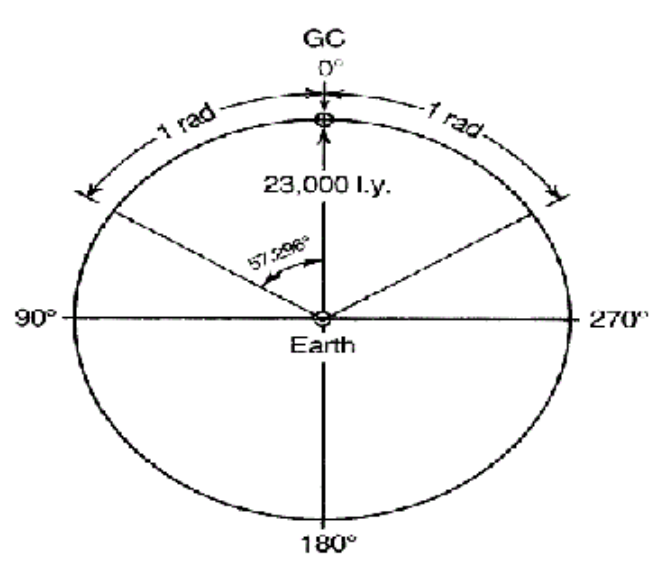

Figure 1: Illustration of the one-radian markers relative to the galactic center (GC), as viewed from Earth.

Such one-radian markers have the advantage that they not only explicitly refer to the Galactic center but also employ a method that indicates that the beacon is intelligently situated, i.e., that its creators have a knowledge of geometry, and that they make reference to our particular viewing location in the Galaxy. That is, these particular longitude sky positions only have significance as Galactic center markers when viewed from our particular Galactic neighborhood location. The same would be true if a marker beacon were placed at the Galactic anticenter location. Such marker locations have the advantage of informing the recipient civilization that a beacon placed at these locations is likely an extraterrestrial intelligent communication signal and not a natural stellar source. In all of these cases, a beacon placed at these locations would be intending to preferentially target our solar system or local group of stars, possibly using a stationary radiation beam.

What do we find at these two one-radian marker locations? Consider the southern GC radian point $\left(\ell=302.648^{\circ}, \mathrm{b}=0^{\circ}\right)$. The pulsar whose sky position comes closest to this location is one that is found at $1=302.637^{\circ}, b=-0.866^{\circ}$, hence deviating by around $0.87^{\circ}$ from this location. It has a period of average length ( $\sim 0.2$ seconds $)$ and no unusual distinguishing features. Of the known pulsars, which currently number to be $\sim 2400$, about $60 \%$, or 1440 , lie within $\pm 5^{\circ}$ of the galactic plane in the longitude range from $\ell=270^{\circ}$ through $\ell=0^{\circ}$ to $\ell=90^{\circ}$. Hence for a pulsar without any unusual characteristics it would not be unusual to find one coming within $0.87^{\circ}$ of this southern point; i.e., (2.37 square degrees / 1800 square degrees $) \times 1440=1.9$. However it is interesting to note that the longitude coordinate for this pulsar deviates by just $0.011^{\circ}$ from the southern one radian point longitude meridian. The odds of this happening due to random chance are less probable, one chance in 11; i.e., $0.011 / 180 \times 1440=0.088$. Again, this is not enormously improbable, but perhaps it is worth paying attention to.

Now consider the northern GC radian point $\left(\ell=57.240^{\circ}, \mathrm{b}=0^{\circ}\right)$. The pulsar that comes closest to this point is PSR $1937+21$, the wellknown Millisecond Pulsar. It is located at $\mathrm{l}=57.509, \mathrm{~b}=-0.290$ and comes within $0.4^{\circ}$ of this one-radian point. Also its longitude coordinate comes within $0.27^{\circ}$ of this one-radian point longitude. If this were a pulsar that had no particular distinguishing characteristics, there would be a chance of about one out of 2.5 of finding one this close to this one-radian position ( 0.5 square degrees / 1800 square degrees $) \times 1440=0.4$. But, on the contrary, this pulsar is indeed very distinctive which requires that we recalculate its probability. Unlike the pulsars at the other two marker locations we had considered, this pulsar is one that really stands out. It has the following unusual characteristics that distinguish it from the average radio pulsar:

a) It is the second fastest pulsing pulsar, its period measuring 1.5578 milliseconds. (The fastest millisecond pulsar is found in a globular cluster located very close to the Galactic center);

b) At radio wavelengths, it is the most luminous of all 130 known millisecond pulsars. Also it is second brightest millisecond pulsar, exceeded only by a millisecond pulsar that lies 25 times closer to us;

c) It is one of 14 pulsars known to emit giant pulses and when it emits a giant pulse, it becomes the most luminous pulsar in the sky. It has been found to emit giant pulses as bright as 65,000 Janskys, as compared with the Vela pulsar which normally is the brightest pulsar at 5 Janskys. The Millisecond Pulsar giant pulses have brightness temperatures as high as $5 \times 10^{39} \mathrm{~K}$, making this the brightest temperature observed in the universe;

d) It is one of only 5 pulsars out of $\sim 2400$ radio pulsars that emit optical pulses and also is the only millisecond pulsar found to emit optical pulses; 
e) It has the lowest proper motion of any pulsar known. Out of 233 pulsars which have had their proper motions determined as of 2005, it has the lowest of all, essentially zero to within the error of observation $(0.8 \pm 2.0$ milliarc seconds per year). This is of interest from the standpoint of this pulsar serving as a position marker because, of all pulsars, the Millisecond Pulsar does not move from the important sky location it appears to be marking.

Each of these unusual characteristics are ones that would tend to get the attention of a recipient civilization, hence making the Millisecond Pulsar qualify as a key marker pulsar. These five rather rare characteristics may be considered as being independent of one another. For example, 42 of 45 glitching pulsars do not exhibit giant pulses and 11 of 14 pulsars that exhibit giant pulses do not exhibit optical pulses. So, given the large percentage of non-correlating pulsar characteristics, one is led to conclude that these characteristics are not causally related to one another. Furthermore, no one has proposed a model that predicts any mutual causal relation between these various characteristics. In the case where there is overlap, for example where a pulsar that undergoes glitching is also observed to exhibit giant pulses, such pulsars are predominantly found to be members of a pulsar subset that appear to be together conveying to us a Galactic message to be described shortly. Employment of more than one unusual behavioral characteristic on the same pulsar would be logical from the standpoint of attracting the attention of the message recipient to this subset of pulsars.

One may ask, what is the probability that a pulsar would have all these unusual characteristics and also come this close to marking this particular Galactic location? The probability for a pulsar to have characteristic (a) is about one out of 1200 , the probability to have characteristic (b) is one out of 130 , the probability to have characteristic (c) is one out of 171 , the probability to have characteristic (d) is one out of 480 , and the probability to have characteristic (e) is one out of 233. Assuming that these characteristics are uncorrelated with one another, we can multiply their probabilities to figure the rarity of having a pulsar with all of these unusual characteristics. This calculates to be $1.3 \times 10^{-13}(0.4 \times 1 / 1200 \times 1 / 130$ $\times 1 / 171 \times 1 / 480 \times 1 / 233)$. So the chance that a pulsar has all these unique characteristics and is located at this particular sky location estimates to one chance in 7.5 trillion!

Even if this method of figuring the statistics is off by several orders of magnitude, most would agree that finding such a unique pulsar at this particular sky position that has ETI communication significance, particularly from our Galactic viewing perspective, is quite extraordinary. Hence, it is worth paying attention to as a possible example of an artificially engineered beacon. On the other hand, one who is unwilling to consider the seven assumptions listed in Table 2 as a context for its interpretation may be left to conclude that this is one of Nature's statistical flukes, one that should be entered into the Guinness Book of Records.

The Millisecond pulsar is discussed here first, because it constitutes the clearest and easiest to understand proof of the pulsar-ETI hypothesis. But, it should be pointed out that the Millisecond Pulsar was discovered after the author had already formed his hypothesis that pulsars may be beacons of ETI origin and that certain of these appear to make reference to the Galactic center.

\section{Related Pulsars in the Message Subset}

If we choose to agree that the marking of this particular sky position is not a highly improbable fluke of Nature, that the very unusual Millisecond pulsar might instead be a beacon engineered by an intelligent civilization (or civilizations) in the Galaxy to intentionally mark this key location which has extraterrestrial communication significance, then we are ready to consider other unusual siting's of distinctive pulsars which together with the Millisecond pulsar appear to synergize with one another to compose a symbolic message. The other pulsars in this communicating subset include the "Vulpecula pulsar" (PSR1930 + 22), the Eclipsing Binary Millisecond pulsar (PSR1957 + 20), the Vela pulsar, the Crab pulsar, and pulsar $0525+21$.

The Vulpecula pulsar is the closest pulsar to the Northern oneradian longitude meridian, deviating by just $0.1^{\circ}$ of arc from galactic longitude $57.2958^{\circ}$. Its position is collinear with the Millisecond Pulsar and the star Gamma Sagittae (Figure 2). Of stars that are visible to the naked eye, Gamma Sagittae, a 3.5 magnitude star that marks the Arrow's tip, is the one that has the closest sky position to the Galaxy's northern one-radian point longitude meridian. Consequently, it is a natural benchmark location for referencing the Galactic center. The trajectory from the Vulpecula pulsar, through the Millisecond Pulsar position currently projects within $0.01^{\circ}$ of arc of Gamma Sagittae and would have been in exact alignment about one millennium ago. A $0.01^{\circ}$ deviation would have odds of one in 18,000 if due to chance. If this alignment is instead interpreted as being intentional, it would imply that the sending civilizations knew the positions of stars visible from our particular viewing perspective that would be bright enough to be easily seen by our astronomers or sky gazers. This implies that the sending civilizations either had traveled to our location sometime in the past and learned the layout of the stars visible in our sky, or that they have a highly accurate astronomical measurements of the locations and relative distances of stars in the Galaxy and use of a highly sophisticated computer model capable of projecting how the starry sky would look from our particular location in the Galaxy.

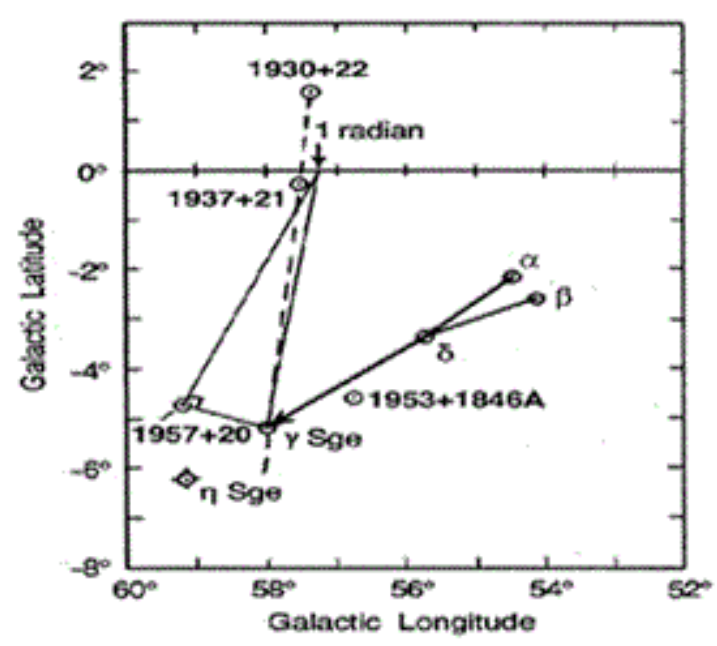

Figure 2: A sky diagram showing the location of the millisecond pulsar (PSR $1937+21$ ), eclipsing binary millisecond pulsar (PSR $1957+20$ ), and the vulpecula pulsar (PSR $1930+22)$ relative to the stars that form the constellation of Sagitta, the celestial arrow. 


\section{(10.4172/2332-2519.1000148}

Page 7 of 13

The Eclipsing Binary Millisecond (EBM) pulsar, or "Black Widow pulsar" as it is commonly known, is another that further calls our attention to the Galaxy's northern one-radian point. This radio pulsar is unusual in that its pulse period comes closest of all pulsars to that of the Millisecond Pulsar, deviating by just $3.184 \%$, and yet it has a sky location just $4.5^{\circ}$ away. This alone beats the odds of random probability. To be the one pulsar that has a period closest to that of the Millisecond Pulsar, the chances are one in $\sim 2400$. The chance of a pulsar lying only $4.5^{\circ}$ away from the Millisecond Pulsar yields one chance in 28 ; i.e., $\Pi \times(4.5)^{2} /\left(180^{\circ} \times 10^{\circ}\right)$.

Furthermore the EBM pulsar is also distinctive in that it is one of only 14 eclipsing binary millisecond pulsars. It is also the fastest pulsing millisecond pulsar of all eclipsing binary pulsars and in addition has the most circular binary orbit of all. Its orbit deviates from perfect circularity by less than one part in a billion! To date no one has offered an explanation as to how such perfect circularity could be achieved by natural means. Moreover because it is eclipsing, its orbital plane is aligned approximately in the direction of our solar system. So the chances of a pulsar being part of a binary system and having these unique orbital characteristics are also one in 2400. In addition, the EBM pulsar is unique in that it is one of only 14 pulsars known to emit giant pulses, which has a chance probability of one in 171 . The probability of a pulsar having all of the above unique characteristics of the EBM pulsar figures to be one in 28 billion; i.e., $1 /(2400 \times 28 \times$ $2400 \times 171)$.

The galactic longitude of the EBM pulsar deviates from the longitude of the Millisecond Pulsar by an amount $\mathrm{BC}=1.68807^{\circ}$ (Figure 3). Dividing this by the angular deviation of the Millisecond Pulsar from the Galaxy's northern one-radian point, $\mathrm{AB}=0.26895^{\circ}$, yields the ratio 6.2765 , very close to the $2 \Pi$ ratio 6.2832 . Due to the proper motion of the EBM pulsar, the ratio would have been exactly 2 $\Pi$ around 1775 C.E. Thus using the Millisecond pulsar longitude as a reference (point $B$ ), this near $2 \Pi$ ratio of $B C$ to $A B$ reiterates the oneradian marker theme, $\mathrm{AB}$ symbolizing the radius of a circle and $\mathrm{BC}$ symbolizing the circle's circumference, This cryptographic check mechanism ensures that we have correctly interpreted the symbolism of the cipher's key, the Millisecond pulsar. Watzlawick [25] explains the importance of using an anticryptographic key and check mechanism in the design of extraterrestrial communication messages, a technique we employed in the Pioneer 10 space plaque message. The EBM pulsar becomes the appropriate designator of the circle circumference concept since its binary orbit is unusually circular, in fact the most circular of any known binary pulsar. Thus these two pulsars with closely matched periods, the Millisecond Pulsar and the EBM pulsar, synergistically reinforce the one-radian symbolism previously inferred for the Millisecond Pulsar. It is expected that an extraterrestrial communication message would make use of geometrical relationships such as this since geometry is a universal language that should be understood by all Galactic civilizations.

The current sky position of the EBM pulsar deviates just $0.0018^{\circ}$ from the galactic longitude position that would allow the $\mathrm{BC}$ chord to make a perfect $2 \Pi$ ratio with $\mathrm{AB}$. For any pulsar to come this close to this position, the odds are one chance in 42 , i.e., $(0.0018 / 180) \times 2400$ $=0.024$. For this particularly unique eclipsing binary millisecond pulsar to be so located, one having the unusual characteristics mentioned above, the odds are one in 1.1 trillion $\left(1 /\left(2.75 \times 10^{10} \times\right.\right.$ 42)). When this is multiplied with the probability figured earlier for the siting of the Millisecond Pulsar, one obtains odds of one chance in about $10^{25}$.
There is also the additional observation that the sky position of the EBM pulsar is such that trajectories drawn from it to the Galaxy's northern one-radian point and also to the star Gamma Sagittae make an almost perfect $90^{\circ}$ angle, deviating by just $0.15^{\circ}$ of arc. We have not included the odds of this in our calculation. But hopefully odds as small as one in $10^{25}$ is sufficient to make the point in favor of the ETI interpretation. Some may disagree with the particular numerical values obtained here for these odds. But regardless of how they are figured, the odds are incredibly small that the Millisecond Pulsar and Eclipsing Binary Millisecond pulsar would have such unique attention-getting characteristics and be making these particular geometrical alignments. Moreover the one-radian symbolism that they convey is relevant to their location.

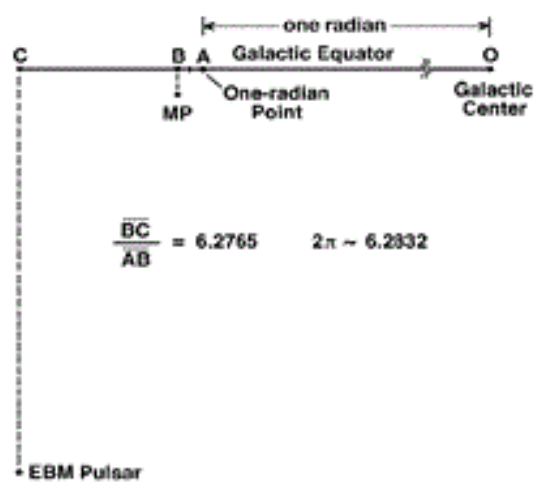

Figure 3: Positions of millisecond pulsar and EBM pulsar in the vicinity of the galactic one-radian point showing how the layout of their longitudes depicts the pi ratio.

The Crab and Vela pulsars also stand out as being quite unique. The Crab pulsar lies $6,585 \pm 200$ light years from us at a sky location that lies at galactic coordinate $\left(184.56^{\circ},-5.78^{\circ}\right), 7.5^{\circ}$ from the galactic anticenter (Figure 4). It is associated with the Crab nebula, a remnant produced by a supernova that was observed in $1054 \mathrm{CE}$. The Vela pulsar lies just $935 \pm(+26,-55)$ light years from us toward galactic coordinate $\left(263.55^{\circ},-2.79^{\circ}\right)$ and overlain by the Vela supernova remnant which was produced by a supernova that would have been observed about 12,800 years ago. Significantly, of all known 274 supernova remnants having ages less than 105 years, these are the two that lie closest to our Sun.

Also these two pulsars are very distinctive. Like the Millisecond Pulsar, they both have the rare property of emitting optical pulses and giant pulses. Also they both exhibit pulsar period glitches, and both produce pulses also in the X-ray and gamma ray spectral region making them among the most easily detected of all pulsars. Furthermore they are distinctive because of their luminosity and brightness. The Crab pulsar is the most luminous pulsar and is also the brightest pulsar in the sky at optical, $\mathrm{x}$-ray, and gamma ray wavelengths. At radio wavelengths it is the second brightest, exceeded only by the Vela pulsar, which is located 7 times closer. These two pulsars may literally be called the "King and Queen of radio pulsars". 


\section{(10.4172/2332-2519.1000148}

Page 8 of 13

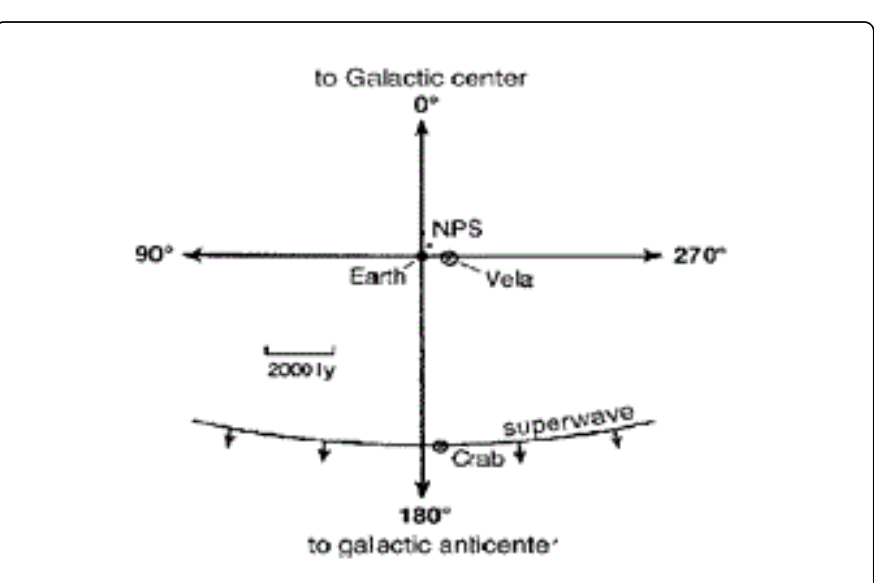

Figure 4: The positions of the Crab and Vela supernova explosions relative to the solar system.

Some may argue that young pulsars are among the more luminous hence that it would be expected that a remnant as young as the Crab pulsar ( 1000 years old) would have a pulsar that would be unusually luminous. This reasoning, however, would not predict that the Crab remnant would harbor the most luminous pulsar in the sky. Nor would it predict that its pulsar should have emission at x-ray and gamma ray wavelengths. Moreover in the case of the Vela pulsar, there is no evidence that it originated from the explosion site of the Vela supernova [26]. It appears merely to be marking the Vela supernova remnant through line-of- sight superposition. Its speed and trajectory indicate that, at the time of the Vela supernova, its sky position was at least 15 light years from the Vela explosion sky position, hence indicating no reasonable association, and implying that its age is largely unknown. Hence it is somewhat surprising that a pulsar with such distinctive pulse characteristics should happen to coincide with or "mark" the closest young remnant to the solar system considering also that it did not originate from the Vela supernova explosion site.

One might ask then, what are the chances of finding two highly unique pulsars and also the most luminous and brightest in the heavens marking these two supernova remnants that lie closest to the Sun? Of the 279 known supernova remnants in the Galaxy, only 54, or about $19 \%$, have pulsars associated with them. So the odds that any one of the two closest supernova remnants would have a pulsar associated with it are about one in five. The odds that radio pulsars would be associated with both of the two closest supernova remnants are one in 25 (i.e., $1: 5^{2}$ ).

But this grossly underestimates the probability of the Crab and Vela pulsar supernova associations. We must consider also the uniqueness of these pulsars. Let us begin with the Crab pulsar. The odds that the Crab nebula would be associated with a pulsar that has the attentiongetting characteristic of being the most luminous radio pulsar in the sky when observed from the radio through the gamma ray part of the spectrum) are one in 60,000 ; i.e., $1 /(5 \times(2400)$. Considering that this pulsar also exhibits a large number of unique attention-getting properties such as optical pulses (observed in $0.44 \%$ of pulsars), giant pulses (observed in $0.62 \%$ of pulsars), interpulses (observed in $1 \%$ of pulsars), and period glitches (observed in about 3\% of pulsars), then the odds that a pulsar would be the most luminous of all, have all of the above unusual features and also coincide with one of these two remnants is estimated at $[1 /(5 \times 2400)] \times 0.0044 \times 0.0062 \times 0.01 \times$ $0.03=1.4 \times 10^{-13}$ or one in 7 trillion.

Vela shares all the same unusual attention-getting features that characterize the Crab pulsar, except that it is instead the third most luminous pulsar. So the Vela pulsar association with the Vela remnant may be calculated in a similar fashion except that it is two times more likely than the Crab pulsar probability. Hence for Vela the odds are one in 3.5 trillion. The odds of both the

Crab and Vela pulsars coinciding with these two nearby supernova remnants, the two that happen to be closest to our solar system, are then about one chance in $2.5 \times 10^{25}$, i.e., $7 \times 10^{12} \times 3.5 \times 10^{12}$. It is this sort of attention-getting coincidence estimated above, one chance in over ten trillion trillion, that leads one to probe deeper to find out whether there might have been some important reason to call our attention to these nearby supernova explosions.

\section{A Possible Message}

My attempt to explain pulsar characteristics in terms of a natural cause first began in 1978 while a doctoral student at Portland State University. This was eleven years after the discovery of the first pulsar, and came at a time when some 300 pulsars had been discovered. As often is the case, new discoveries are made after the data has already been collected and it is only afterward that a pattern is recognized in that data. It was in 1979 that I noticed the improbability of the alignment of the Crab and Vela pulsars with the two supernova remnants which of all young remnants happen to be the closest to our solar system. I also noted that the explosion dates for the Vela and Crab supernova were temporally separated by the amount of time it would take for a radiation wave or light echo to travel outward from the Galactic center toward the anticenter, reaching first the Vela explosion site and then the Crab explosion site. Less than a year prior to this I had discovered that certain ancient constellation lore indicates that our Galaxy's core had produced a Seyfert-like outburst near the end of the last ice age, an event that would have been observed around the time that the Vela supernova is believed to have occurred [27]. It was in this context that I came to hypothesize that these two pulsars may be purposely engineered beacons and that they were given their observed unusual characteristics to call our attention, not only to these two unique nearby supernova remnants, but also to the galactic core explosion whose radiation wave would have been passing through this region at the time these supernova occurred.

This apparent "message," then led me to infer that both supernovae may have been sequentially triggered by an energetic disturbance associated with this core explosion event, e.g., light echo, cosmic ray volley or gravity wave that propagated radially out through the Galaxy at close to the speed of light, a phenomenon that I termed a "galactic superwave." I hypothesized that this energetic disturbance would have passed through our solar system about 12,150 B.C.E., caused the Vela supernova progenitor star to explode about 11,565 B.C.E. (visible on Earth about 815 years later in 10,750 B.C.E.), and then caused the Crab supernova progenitor star to explode about 5,530 B.C.E. (visible on Earth in $1054 \mathrm{AD}$ ); Figure 4 [27]. Both the superwave concept and the idea that the Galactic core had exploded in recent prehistoric times were novel concepts, and so I chose to investigate their validity as the subject of my Ph.D. dissertation. This investigation also involved testing the hypothesis that our galactic core periodically has a significant effect on the Earth and solar system. The results of this research proved to support this hypothesis [28]. A check of the 
geologic record indicated that at the end of the last ice age, 16,000 to 11,000 years ago, and on other past occasions, the Earth did have a prolonged increase in its beryllium-10 production rate relative to levels prevailing during the Holocene, suggesting a higher than normal cosmic ray exposure at that time [27,29,30]. Also a check of astronomical data indicated a variety of evidence supporting the idea that an energetic cosmic ray / gamma ray volley did radiate isotropically from the Galactic center and pass through the Solar System at about that time, and that this same volley is currently in the vicinity of the Crab Nebula, Tycho, and Cassiopeia A supernova remnants where it is in the process of energizing their emission $[27,28,30]$. In the years that followed, more than 14 predictions of this theory were subsequently confirmed.

Consequently, the Vela and Crab pulsar beacons played a very important role in prompting the formation of this superwave hypothesis. Without these stellar markers calling our attention to these supernova remnants, one would not have as readily formulated the idea that an explosion of our Galaxy's core could penetrate so far through the Galaxy to have affected our galactic neighborhood. Such an idea, indeed, had not previously been considered in the literature. At that time, I hypothesized that these two pulsars may be artificial communication beacons placed at these locations either to refer to, or to alert us to, this energetic wave of Galactic core emission that apparently had passed through our solar system at the end of the ice age and had a decisive impact on our Galactic locale. In fact, one of the largest mammalian extinction events in recent geological times occurred close to this date likely due to a solar cause [31]. The fact that this pulsar message did play a role in the initial formulation of this hypothesis is documented in my dissertation laboratory notebook.

These details of when and how the author had formulated the ETI pulsar-beacon-message hypothesis, i.e., that radio pulsars may be of artificial origin and that a subset of such pulsars appear to refer to the Galactic center, are recounted here to point out that this hypothesis had been established and was in the process of being checking out before the discovery of millisecond pulsars. The Millisecond Pulsar, for example, was discovered in 1982 and the Eclipsing Binary Millisecond pulsar in 1988. Since the Millisecond Pulsar geometrically designates the distance between the Galactic center and the Sun by marking a galactic equator location very close to one radian from the Galactic center, it may be taken as likewise conveying a message that makes reference to both the Galactic center and solar vicinity. Hence the discovery of the Millisecond Pulsar in this particular sky location reinforces and supports the a priori formulated hypothesis that the Vela and Crab pulsars intentionally convey a galactic-core / solarsystem message.

Often researchers may believe they have perceived a pattern in their data and will do an a posteriori statistical analysis of existing observational data to justify the presence of a pattern they believe to be present. It is in such situations that they must take a critical attitude of their own work and exercise caution to avoid fooling themselves. So one might ask, in drawing these conclusions about the Millisecond and EBM pulsars whether the author was projecting the presence of a pattern in what may be inherently random pulsar arrangements. It is true that the initial hypothesis did not lead the author to predict ahead of time that a very unique pulsar might be found at this northern equatorial one-radian location and to inform radio astronomers to look there for a possible beacon. Back in the late 70's when it was discovered that the Crab and Vela pulsar were making reference to a cosmic ray event that propagated all the way from the galactic center to the solar system, the author's efforts were mainly focused on validating the superwave hypothesis. But, if one surmises that the Crab and Vela pulsars are part of a message alerting us to such a superwave, it is not much of a leap of induction to infer that a galactic equator sky location situated one radian from the Galactic center would be very significant in the context of this message, in that it marks the angular distance of a line segment vector that extends between the Galactic center and our solar system. Hence the Millisecond pulsar marker very appropriately fits the previously formed superwave concept.

Moreover, as pointed out earlier (Section 3), it would be logical for an extraterrestrial civilization that intends to message our solar system to place a beacon at this particular galactic sky location due to its specific reference to the Galactic center. Also, a recent Galactic core explosion would be a likely topic for interstellar discussion because core explosions are a phenomenon that all civilizations in our Galaxy would be aware of and, to varying extents, would be affected by. So, it is a relatively minor step to realize that the Millisecond pulsar is also likely of artificial origin and part of this pulsar-galactic-center message. Curiously, of all pulsars the Millisecond Pulsar is the only one, other than the Crab and Vela pulsars, to exhibit both optical pulses and giant pulses, attention-getting features that invite the message recipient (us) to mutually associate these three otherwise unrelated sources (Table 3).

\begin{tabular}{|c|c|c|c|c|c|c|c|}
\hline Pulsar & $\begin{array}{l}\text { Gamma } \\
\text { pulses }\end{array}$ & $\begin{array}{l}\text { X-ray } \\
\text { pulses }\end{array}$ & $\begin{array}{l}\text { Optical } \\
\text { pulses }\end{array}$ & $\begin{array}{l}\text { Giant } \\
\text { pulses }\end{array}$ & Interpulses & $\begin{array}{l}\text { Period } \\
\text { glitches }\end{array}$ & $\begin{array}{l}\text { Ultrashort } \\
\text { period }\end{array}$ \\
\hline Crab & $x$ & $x$ & $x$ & $x$ & $x$ & $x$ & \\
\hline Vela & $x$ & $\mathrm{x}$ & $x$ & $x$ & $x$ & $x$ & \\
\hline Millisecond & & & $x$ & $x$ & & & $\mathrm{x}$ \\
\hline EBM & & & & $x$ & & & $x$ \\
\hline Vulpecula & & & & & & $x$ & \\
\hline PSR0525+21 & & & & & & $\mathrm{x}$ & \\
\hline
\end{tabular}

Table 3: Unusual characteristics of key pulsars. 


\section{The Transient Pulsar GCRT J17445-3009}

There are other pulsars that appear to be part of this message, such as PSR $0525+21$; a glitching pulsar positioned just $1.3^{\circ}$ of arc from the Crab pulsar. This one appears to be pointing out the orientation of our solar system's ecliptic plane in the sky and the angular deviation of the ecliptic plane from the Crab pulsar sky position; LaViolette [11].

Another radio source that may be an artificial beacon calling our attention to the Galactic center is the transient radio pulsar GCRT J17445 - 3009. This source was observed on three occasions between September 2002 and March 2004, and since that time has not been seen again [32]. This one may be considered a candidate for several reasons. First, it was unusually bright. When it was observed between September 2002 and September 2003, it shone with a radio brightness of 1.67 Janskys making it the second brightest pulsed radio source in the sky, exceeded only by the Vela pulsar. Its brightness temperature was estimated at about $10^{16} \mathrm{~K}$ implying an energy density vastly exceeding those of most other classes of radio sources [32]. This led its discoverers to conclude that it was beaming coherent emission to us. Second, as a transient source, it was very unusual since no other transient radio source has been observed to produce pulses with such regularity, unless one wishes to interpret it as an extreme nulling pulsar as have Zhu and $\mathrm{Xu}$ [33]. A third unusual feature is its unusually long period of 77 minutes. The longest period radio pulsar has a period of 11.7 seconds; so this one has a period that is longer by a factor of 395 . Fourth, the radio pulse of the transient source spanned 10 minutes, or 13 percent of its pulse period. Few pulsars exhibit a pulse that spans such a large fraction of its pulse period. Others that do so include the Crab pulsar, the Vela pulsar, and about three others. A fifth feature that prompts one to single this one out is that the sky position of this source lies so close to the Galactic center, just $1.1^{\circ}$ of arc southwest of the Sgr $\mathrm{A}^{*}$, and it lies about the same distance away as the Galactic center. Since the time that it was discovered, only two pulsars have been found which have sky positions closer to the GC by about 8 minutes of arc. But no unusual characteristics have been reported that would make them stand out in a similar way.

The probabilities for this pulsar calculate to be $2 / 2400$ that it would be the second brightest radio pulsar, $1 / 2400$ that it would have the longest pulsar period, 6 / 2400 that it would be a pulsar whose pulse occupies a substantial fraction of its pulse period, and 3 / 2400 that it would be the third closest to the Galactic center. All together, the chances of having a pulsar with all of these unique characteristics figures to about one chance in a trillion.

Prior to the discovery of this source it was proposed that subsets of radio pulsars, those discussed above, are making explicit reference to the Galactic center [12]. So, the subsequent finding of this very unique transient source positioned so close to the Galactic center sky position, strengthened this hypothesis and led the author to conclude that this pulsar too may likely be a beacon intentionally placed to call our attention to the Galactic center [11]. Although, this source has remained radio quiet since the time of its discovery.

\section{A Technology for Producing a Pulsar-like Communication Beam}

One method of astro-engineering pulsar emissions presupposes that a civilization has developed the ability to journey close to a cosmic ray emitting neutron star or X-ray star. Once positioned in its vicinity and properly shielded from its cosmic ray flux, a properly equipped spaceship could project a maser beam to an ionized region in the star's corona and phase conjugate microwaves reflected back to the maser to produce a self-amplified, phase conjugate soliton beam between the ship's maser generator and the star's corona $[11,12]$. Such microwave phase conjugate resonator technology has been developed by the military mainly for use as a directed energy weapon or for space propulsion [21], although its application to radar signal amplification has also been explored [34].

By interfering properly chosen microwave frequencies, it is possible to create a magnetic field grating pattern in the star's coronal region that would locally decelerate its outgoing relativistic electron (and proton) flux and produce an outward directed synchrotron beam. Due to the relativistic beaming effect, this emitted radiation would be confined to a forward-directed conical beam having an aperture of $2 /$ $\gamma$ radians and a luminosity that would scale as $8 \gamma 3$, where $\gamma$ is the Lorentz factor of the cosmic ray electrons. The Crab pulsar neutron star is estimated to emit cosmic ray electrons having Lorentz factors of up to $\gamma \sim 10^{7}$. In this case, the generated synchrotron beam would have a divergence of $\sim 10^{-7}$ radians, which would produce a beam target diameter of about $40 \mathrm{AU}$ at a distance of 6500 light years.

By modulating the magnetic field intensity of the grating pattern, the communication beam intensity may be modulated in periodic fashion to produce highly precise, attention getting pulses. Grating fields' oriented perpendicular to the outgoing cosmic ray flux would generate linearly polarized synchrotron emission while fields oriented parallel to the outgoing flux would be effective in generating circularly polarized emissions. Through proper manipulation of this remote field grating pattern, pulsed signals could be produced having complexities comparable to those observed to come from pulsars.

\section{Synchrotron Particle Beam Communicators vs. Phased Array Antenna Communicators}

The idea of using stationary broadband radiation beams targeted on distant stellar locations for interstellar communication purposes, initially proposed by the author $[10,12]$, has more recently received indirect support from the ideas presented by the Benford group. Benford et al. [35,36] have shown that there is an economical advantage in using stationary targeted broadband electromagnetic beams for communication with extraterrestrial intelligence (CETI) in that far less power and operating cost is required to send one's signal as compared with more traditional discrete-frequency, non-directed radio transmissions.* As an example of how such a communicator beam might be constructed, they propose using a deployed array of microwave antennas fed by high-power gyrotrons and networked to operate as a phased array so as to emit a pulsed microwave beam in a particular direction. In one of their examples, they consider a $5.1 \mathrm{~km}$ diameter antenna array consisting of 3000 gyrotrons, each capable of emitting a few megawatts of power. They figure a total power requirement of $6.9 \mathrm{GW}$ and antenna gain of $\sim 10^{9}$ to produce an effective isotropic radiated power (EIRP) of $10^{19}$ watts. This is emitted as a beam having an angular divergence of about $10^{-4}$ radians which is able to communicate over a distance of $\sim 6000$ light years and illuminate a region about 1 light year in diameter. They propose transmitting at a frequency of $\sim 1 \mathrm{GHz}$ and estimate a bandwidth of about 1 megahertz for the oscillator power levels they consider, the limited frequency spread of their beam being determined by the physics of microwave oscillation in gyrotron cavities.

Ten years earlier a substantially different method was proposed for a "low-tech" terrestrial- based device capable of producing a targeted 
interstellar communication beam, one that involved modulating the output of a free-electron maser [11,12]. In this design, a linear accelerator would generate a continuous beam of ultra-relativistic electrons which, in turn, would be directed into a wiggle field modulator consisting of transverse magnetic fields generated by a series of superconducting magnets. These wiggle fields would cause the electrons to emit a beam of synchrotron radiation. Another version of the device configures the imposed magnetic field lines parallel to the particle trajectory so as to produce an electron cyclotron maser. In either version, due to the relativistic beaming effect, this emitted radiation is confined to a forward-directed narrow conical beam having an aperture of $2 / \gamma$ radians and a luminosity that would scale as $8 \gamma 3$, where $\gamma$ is the Lorentz factor of the cosmic ray electrons. By varying the power to the imposed magnetic fields, the communication beam intensity may be modulated in periodic fashion to produce highly precise, attention-getting pulses.

The radiation beam of this communicator was proposed to have a median frequency of $f=400 \mathrm{MHz}$ and bandwidth of $\Delta f=400 \mathrm{MHz}$ and an intensity sufficient to produce a 0.8 Jansky signal at a distance of 6500 light years, hence a signal strength equal to that coming from the Crab pulsar. Calculations indicate that this would require a particle accelerator capable of producing a 1 megawatt beam of $50 \mathrm{Gev}$ electrons having a Lorentz factor of $\gamma=2 \times 10^{5}$. The particles would produce a synchrotron beam whose divergence calculates to be $10^{-5}$ radians, which yields a beam diameter of about 0.065 light years (4 $\mathrm{AU})$ at a target distance of 6500 light years provided that the particle beam cosmic rays have trajectories that are initially parallel to one another. Choice of a lower $\gamma$ for the particle beam increases the beam divergence and reduces the Doppler boosting of the synchrotron emission but necessitates an increased relativistic particle energy flux to maintain equivalent target signal intensity. Conservatively estimating an efficiency of $1 \%$ for converting electric power to relativistic electron power and in turn into synchrotron beam power indicated that such a communicator beam would require an input power of $100 \mathrm{MW}$. So this version would use about 70 times less power than what the Benfords estimate for their phased array communicator and would produce a beam having a 50 fold lower EIRP.

The cost for constructing the proposed particle beam communicator should not be much more than $\$ 5$ billion, similar to the cost of building the Large Hadron Collider. Using an input power of $120 \mathrm{MW}$, the Large Hadron Collider generates $70 \mathrm{Gev}$ electrons (Lorentz factor $\gamma$ $\sim 2 \times 10^{5}$ ), hence comparable to this communicator example. One must consider that the cost of accelerator technology is progressively dropping over the years with the advent of beat wave and wake field accelerators used in producing free electron lasers. So the construction cost could be substantially less than estimated here. Certainly, it would be an order of magnitude less than the $\$ 41$ billion that the Benfords estimate for their 6000 light-year pulsed beam communicator.

The Benfords refer to their phased array communicator as broadband, as distinguished from the prior notion of discrete frequency communication. The author also had used the term broadband to refer to the pulsar particle beam communicators [12]. However, these synchrotron beacons would produce a much broader frequency spread than those that the Benfords have considered $(\Delta \mathrm{f}>$ $100 \mathrm{MHz}$ as compared with $\Delta \mathrm{f} \sim 1 \mathrm{MHz}$ ). Hence compared to the broadband beams of the Benfords, these particle beam beacons would be ultra-broadband. It is generally agreed that a beacon that spans a greater bandwidth is desirable from the standpoint of ETI communication since this increases the chance of the beacon being detected by a target civilization.

Use of a natural cosmic ray source such as a neutron star, instead of a fabricated particle accelerator, to power a synchrotron beam communicator has the advantage that one would have a much greater spread of relativistic particle energies and hence would necessarily produce a much broader spectrum synchrotron beam spanning many decades of the radio frequency spectrum and in some cases could extend up to optical X-ray, and gamma ray wavelengths. Compared to the more "low-tech" earth-based particle accelerator communication beam technology, this star- engineered beam technology would have a much lower energy demand. An initial investment of energy would be needed to power up the maser phase conjugate resonator to create the projected field grating pattern. But once the phase-conjugate beam was activated, no additional energy would be needed to be added to it since the soliton beam would be able to entrain energy from the impinging stellar cosmic ray flux. That is, energy from the outgoing cosmic rays would transfer to the maser soliton beam within the wiggler field grating pattern established in the star's corona, thereby allowing the maser beam to become self-powered. Also it is characteristic of phase conjugate resonators that they confine their maser flux to their resonator beam which produces relatively low energy losses. This beam technology may even have a secondary use for deriving useful energy from the tapped neutron star.

Many such stationary pulsed synchrotron beams could be produced and pointed in various directions away from a single neutron star to continuously target a large number of Galactic locations. This would avoid the need to retarget a single beam to a series of different locations in a repeating cycle as the Benfords' technique requires. Consequently, we would be aware only of the existence of those beams being targeted directly towards us. What we have detected as pulsar signals may be beams being aimed in the direction of our Galactic neighborhood.

Assuming that a phased microwave antenna array would be the only way a civilization would be able to generate a targeted broadband communication beam, the Benfords [37] have chosen to interpret pulsars as natural sources and have advised that SETI investigators should avoid confusing pulsar signals with ETI communication sources. They state their belief that pulsars are "clearly radiation from rotating star magnetospheres." However, as mentioned earlier, a large number of pulsar astronomers do not take such a confident stance about the explanatory power of the lighthouse model. As a conceptual model it is useful for discussing and publishing various pulsar observations, but many astronomers are far from satisfied with it.

As one example, the lighthouse model is easily disproved in the case of the Crab pulsar. The Crab pulsar has a pulse that spans a large portion of its period, but it also exhibits an interpulse about $145^{\circ}$ after the main pulse. Pulsar modelers have attempted to explain the first feature as indicating that the pulsar is aiming its pole towards us and precessing its hot spot around this axis to create an extended pulse. But they attempt to explain the second feature by assuming that the main pulse and interpulse come from opposite poles of the neutron star and that the pulsar's axis is spinning end-for-end relative to our line of sight so that first one pole and then the other shines in our direction. Since these interpretations are incompatible with one another, one is led to conclude that the lighthouse model explanation for the Crab pulsar is in need of revision. 
Also the lighthouse model has difficulty accounting for the off-pulse constant background emission observed in pulsars PSR B0525 + 21 and PSR B2045 - 16. In both of these pulsars radio synchrotron emission has been detected at a pulse phase angle far from the main pulse $[38,39]$. The pulse profile is typically sampled in the off-pulse phase range from $80^{\circ}$ to $250^{\circ}$ from the center of the main pulse, the main pulse spanning about $20^{\circ}$ of the $360^{\circ}$ pulse phase. This low level emission prevails as a residual beamed flux that is emitted in between the pulsar's main pulses at $5 \%$ of the main pulse intensity in PSR B0525 +21 and $1.5 \%$ of the main pulse intensity in PSR B2045 - 16 . Observation has shown that the off-pulse emission has about the same spectral index as the main pulse emission and hence must originate from the pulsar's immediate vicinity as coherent beamed radio emission [39]. Hence emission from a pulsar wind nebula has been ruled out. This phenomenon cannot be explained as emission coming from the pulsar's magnetic pole because during this off-pulse interval its pole would be expected to point away from the observer. Consequently, the lighthouse model has a difficult time accounting for this. A beacon projecting a stationary targeted beam, however, could produce this residual emission simply by decreasing the magnetic field intensity in the artificially generated grating pattern and thereby diminishing the intensity of the emitted synchrotron flux to a much lower level.

The Benfords state that one reason radio pulsars should not be considered as ETI beacons is that they have a very large bandwidth of at least $400 \mathrm{MHz}$ and that this is far larger than the $1 \mathrm{MHz}$ bandwidth limit imposed by the physics of their phased array antenna technology. They also suggest that a communicating civilization would attempt to produce a signal that is not pulsar-like to ensure that their transmission is not mistaken as coming from a natural source. If anything, this illustrates the point made earlier that the assumptions that one holds, or paradigm that one adheres to in regard to CETI capabilities plays a major role in one's decision as to whether an observed source might be or might not be of ETI origin. One may conclude that this decision to rule out pulsars is rooted in restrictive views as to the particular technologies that are available for broadband communication.

Four years after the author had noted the possible ETI nature of radio pulsar GCRT J17445 - 3009 [11], Benford et al. [36] also noted the unusual nature of this source and discussed whether or not it might be of ETI origin. They concluded that it was likely not an engineered signal because it would cost too much for an alien civilization like our own to build a powerful enough transmitter. Here they consider that the transmitting civilization would use a phased array antenna technology similar to what they propose. They concluded that an antenna array large enough to produce the observed 1.67 Jansky radio signal intensity from a distance comparable to a Galactic center distance of 26,000 light years would cost about $\$ 20$ trillion and note that even if the transmitter were located as close as 1000 light years, the cost would still be a prohibitive $\$ 730$ billion.

However, analysis of records of the GCRT J17445 - 3009 radio bursts indicate that their coherent emission was predominantly circularly polarized [40]. This fits with the particle beam communicator model suggested here in which the braking magnetic field is aligned parallel to the cosmic ray electron flux, since the resulting electron cyclotron maser emission would be characterized by a high degree of circular polarization. Beamed emission from a phased antenna array, however, would have a rather small circular polarization component since any circular polarization produced by adjacent microwave horns in the antenna array would oppose and cancel out one another. So there is no reason to rule out the ETI nature of this source on the basis of a hypothetical phased array antenna technology.

\section{Conclusion}

As discussed in Section 3, besides the Galactic center, there are three principle sky positions viewable from our solar system where a civilization could site a communication beacon and be assured that their signal would be interpreted as an ETI message through its reference to the Galactic center, these being the galactic anticenter location and the northern and southern locations along the galactic equator that lie one radian from the Galactic center. One of these, the Galaxy's northern one-radian point is clearly and unambiguously marked by an astoundingly unique source. The unusually low chance probability of finding a pulsar as unique and attention getting as the Millisecond Pulsar so close to the position that lies one-radian from the Galactic center should by itself give strong support to the interpretation that this pulsar in particular is an interstellar communication beacon. But in addition, there are several other unusual pulsars in this vicinity which synergistically reinforce this interpretation. When considered together with the strategic placement of the highly unique Crab and Vela pulsars which share many of the same unique characteristics as the Millisecond Pulsar, the conclusion that they are communicating a message involving reference to the Galactic center becomes even more convincing. Besides this there is the temporary appearance of the radio pulsar transient source GCRT J17445 - 3009 which also raises interest because of its close proximity to the Galactic center.

We are therefore led to conclude that these subsets of pulsars, as well as other radio pulsars, are artificial sources, and that Jocelyn Bell's first hunch was indeed the correct one. Of course, those who choose not accept the ETI hypothesis and who also feel uncomfortable in accepting the improbability of a natural explanation, always have the third option: namely that Nature is itself intelligent and is trying to communicate with us.

\section{References}

1. Patruno A, Watts AL (2012) Accreting millisecond X-ray pulsars, arXiv: 1206.2727.

2. Jonker PG, Torres MAP, Steeghs D (2008) Optical and X-ray observations of IGR J00291 + 5934 in quiescence. Astrophysical Journal 680: 615-619.

3. Filippenko A, Radhakrishnan V (1982) Pulsar nulling and drifting subpulse phase memory. Astrophysical Journal 263: 828-834.

4. Bartel N, Morris D, Sieber W, Hankins TH (1982) The mode-switching phenomenon in pulsars. The Astrophysical Journal 258: 776-789.

5. Taylor JH, Manchester RN, Huguenin GR (1975) Observations of pulsar radio emission. I. Total-intensity measurements of individual pulses. The Astrophysical Journal 195: 513-528.

6. Gil J, Krawczyk A, Melikidze G (1997) Pulsar radiation. Banach Center Publications 41: 239-255.

7. Gil J, Melikidze G, Zhang B, Geppert U, Haberl F, et al. (2007). Drifting subpulse phenomenon in pulsars: Lofar perspective. Lofar Workshop, April 2007.

8. Hermsen W, Hessels JWT, Kuiper L, van Leeuwen J, Mitra D, et al. (2013) Synchronous X-ray and radio mode switches: A rapid global transformation of the pulsar magnetosphere. Science 339: 436-439.

9. Andersson N, Glampedakis K, Ho WCG, Espinoza CM (2012) Pulsar glitches: The crust is not enough. Phys Rev Lett 109: 241103. 
10. LaViolette PA (2000) Evidence that radio pulsars may be artificial beacons of ETI origin. Proceedings of the 195th meeting of the American Astronomical Society, Session 33, January 12, 2000.

11. LaViolette PA (2006) Decoding the Message of the Pulsars. Bear \& Co. Rochester, VT.

12. LaViolette PA (2000) The Talk of the Galaxy. Starlane Publications, Alexandria, VA.

13. Strom RG (2015) We are not alone: Extraterrestrial Technological Life in our Galaxy. Astrobiol Outreach 3: 144

14. LaViolette PA (2014) Autopoietic gene-enzyme cycles and the emergence of life. J Biol Syst 22: 393-412.

15. Wickramasinghe NC (2015) Evidence to clinch the theory of extraterrestrial life. Astrobiol Outreach 3: e107.

16. Wickramasinghe C, Wainwright M (2015) Convergence to cometary panspermia: Time for disclosure? Astrobiol Outreach 3: 145.

17. LaViolette PA (1990) Galactic core explosions and the evolution of life. Anthropos 12: 239-255.

18. Carot A, Aichmann H, Nimtz G (2012) Giant negative group time delay by microwave adaptors. Europhysics Letters 98: 64002 .

19. Enders A, Nimtz G (1992) On superluminal barrier traversal. Journal de Physique I 2:1693-1698.

20. Nimtz G, Carot A (2012) Actual measurements of superluminal communications via electromagnetic waves propagating within special guides. International Conference of Numerical Analysis and Applied Mathematics, Kos, Greece.

21. LaViolette PA (2008). Secrets of Antigravity Propulsion. Bear \& Co., Rochester, VT.

22. Podkletnov E, Modanese G (2011) Study of light interaction with gravity impulses and measurements of the speed of gravity impulses. In: Modanese G and Robertson R (Eds.) Gravity-Superconductors Interactions: Theory and Experiment. Bussum, Netherlands: Bentham Science Publishers.

23. Podkletnov E, Modanese G (2003) Investigation of high-voltage discharges in low pressure gases through large ceramic superconducting electrodes. J Low Temp Phys 132: 239-259.

24. Walkowicz L, Turner EL, Meadows V, Scargle J, and Zee A (2012) Stellar lighthouses: Decoding signatures of advanced civilizations in precision stellar photometry. John Templeton Foundation International Grant and Essay Competition.
25. Watzlawick P (1976) How Real Is Real? Vintage Books, New York.

26. Bignami GF, Caraveo PA (1988) On the birthplace of PSR 0833-45: Or, is the Vela pulsar associated with the Vela SNR? Astrophysical Journal 325: L5-L7.

27. LaViolette PA (1987) Cosmic ray volleys from the Galactic center and their recent impact on the Earth environment. Earth, Moon, and Planets 37: 241-286.

28. LaViolette PA (1983) Galactic Superwaves and Their Impact on the Earth Environment. Schenectady, Starlane Publications NY.

29. LaViolette PA (1985) Evidence of high cosmic dust concentrations in Late Pleistocene polar ice. Meteoritics 20: 545-558.

30. LaViolette PA (2005) Earth Under Fire. Bear \& Co., Rochester, VT.

31. LaViolette PA (2011) Evidence for a solar cause of the Pleistocene mass extinction. Radiocarbon 53: 303-323.

32. Hyman SD, Joseph T, Lazio W, Kassim NE, Ray PS, et al. (2005) A powerful bursting radio source towards the Galactic Centre. Nature 434: 50-52.

33. Zhu WW, Xu RX (2014) GCRT J1745-3009: A precessing radio pulsar? Monthly Notices R Astron Soc Let 365: L16-L20.

34. Tang R, Lee KM, Chu R, and Howard GL (1993) Radar cross section enhancement using phase conjugated impulse signals. US5223838 A, assigned to Hughes Aircraft.

35. Benford J, Benford G, Benford D (2010) Messaging with cost optimized interstellar beacons. Astrobiology 10: 475-490.

36. Benford J, Benford G, Benford D (2010) Searching for cost optimized interstellar beacons. Astrobiology 10: 491-498.

37. Benford J (2010) How can we distinguish transient pulsars from SETI beacons? arXiv:1003.5938.

38. Basu R, Athreya R, Mitra D (2011) Detection of off-pulse emission from PSR B0525+21 and PSR B2045-16. Astrophysical Journal 728: 157.

39. Basu R, Athreya R, Mitra D (2012) On the nature of Off-pulse emission from pulsars. Astrophysical Journal 758: 91.

40. Roy S, Hyman SD, Pal S, Joseph T, Lazio W, et al. (2010) Circularly polarized emission from the transient bursting radio source GCRT J17445-3009. Astrophysical Journal Letters 712: L5-L9. 